\title{
A MATTER-ANTIMATTER MODEL FOR QUASI-STELLAR OBJECTS
}

\author{
AINA ELVIUS \\ Stockholm Observatory, Sweden
}

\begin{abstract}
Observations of quasi-stellar objects and radio galaxies indicate that the total energy radiated from such objects is so large that the most likely source of energy is annihilation.

The demand for symmetry in the universe between ordinary matter and antimatter indicates that there must be equal quantities of the two kinds of matter in every galaxy. From this it seems likely that a galaxy is born as an ambiplasma body, in which separation of matter from antimatter leads to reasonably stable configurations.

The violent events observed in quasi-stellar objects are then interpreted in terms of collisions between stars of opposite kinds of matter. Such collisions are expected to occur frequently in very young galaxies with a high stellar density in the nucleus. Most of the gamma-radiation released in the annihilation will be absorbed in the gases of the colliding bodies, causing strong heating and violent explosions. Strong ionizing radiation and expanding gas clouds will give rise to the observed optical line emission. Expanding clouds of light ambiplasma will emit synchrotron radiation.
\end{abstract}

\section{Introduction}

The present paper will be concerned mainly with the explosive events occurring in quasi-stellar objects and in the active nuclei of some types of galaxies. General ideas concerning the presence of antimatter in galaxies and the energy release in quasi-stellar objects and in different types of radio galaxies due to the annihilation of matter and antimatter have been outlined in an earlier paper (Alfvén and Elvius, 1969). A 'symmetric cosmology' was adopted, assuming the presence of equal amounts of antimatter and ordinary matter in the universe and in every individual galaxy.

As antimatter cannot be well mixed with ordinary matter without being annihilated in a short time at the densities of interstellar gas in a typical galaxy, the formation of the galaxy from a thinner ambiplasma must have been accompanied by a separation process. Some processes have been suggested (Alfvén and Klein, 1963; Alfvén, 1965), which may cause a separation, resulting in many small cells of pure matter and pure antimatter, later growing into larger regions in which stars can form. Unseparated parts of the ambiplasma will be annihilated. In the present paper a stage of evolution of the galaxy is considered in which the stars have already been formed in great numbers.

\section{Outline of Model}

We assume that the phenomena of quasi-stellar objects occur in certain young galaxies in which the concentration of stars in the central part is unusually high. The observed activity within very small regions of QSOs and of some galaxy nuclei makes such an assumption seem plausible, and similar ideas have been adopted by a number of authors working with various models (e.g. Field, 1964; Colgate, 1969; Low, 1970). In the very dense nucleus collisions between stars will be frequent. On the average, 
in our model every second collision will occur between bodies of opposite kinds of matter which leads to violent explosions. Colliding stars need not be of the same size. Typically a small star or a body of substellar size will fall into a bigger star with a velocity of the order of $1000 \mathrm{~km} \mathrm{~s}^{-1}$. When the bodies collide, annihilation will start immediately, but in order that the velocity of the falling body may be reversed, a fairly large quantity of matter must be annihilated. This requires thorough mixing of antimatter with matter. As such a mixing is a relatively slow process, the smaller body will penetrate the bigger star well below its surface, before most of the annihilation takes place. Most of the gamma-radiation from the annihilation process will then be absorbed in the stellar gas, causing strong heating and shock waves. The consequence will probably be a violent explosion with a release of very large energies, toward which also nuclear reactions in the distorted stars may contribute. Only in favourable cases will the star of smaller mass be entirely annihilated. In less efficient collisions where only a small part of the colliding masses annihilates, the energy due to the explosions of the stars may reach the same order of magnitude as the annihilation energy itself.

\section{Energy Requirements}

We adopt the hypothesis that the quasi-stellar objects are very distant and therefore very luminous. According to recent observations by Kleinmann and Low (1970) the infrared flux from the QSO 3C 273 might be as high as several times $10^{48} \mathrm{erg} \mathrm{s}^{-1}$. This flux may be used as a plausible upper limit for this object, because it is based on an extrapolation of the intensity of infrared radiation actually observed toward longer wavelengths, when it was assumed that the infrared spectrum of $3 \mathrm{C} 273$ is very similar to the spectra recorded for the nucleus of our galaxy and for NGC 1068.

Proton-antiproton annihilation produces neutrinos, gamma-rays, and relativistic electrons and positrons with energies around $100 \mathrm{MeV}$. If the unobservable energy going into neutrinos is not included in the calculations, we find that the annihilation of one solar mass per year will release, on the average, $3 \times 10^{46} \mathrm{erg} \mathrm{s}^{-1}$. The annihilation of matter and antimatter in a QSO required to explain the total energy radiation would thus be approximately in the range from around one solar mass to about a hundred solar masses per year.

\section{Comparisons of Model with Various Observed Properties}

\section{A. SIZE OF SOURCES}

Interferometer measurements (Kellermann et al., 1968) as well as the shape of the radio spectra (Kellermann and Pauliny-Toth, 1969) and observations of rapid intensity variations by numerous investigators, all indicate that the sources in QSOs are often very small and that in several cases more than one source may exist in a QSO. This is in good agreement with our model. The remnants of colliding and exploding stars are certainly small sources and the model is necessarily characterized by strong 
variations in the energy output. Several sources will usually be active at the same time in the nucleus of a QSO, although they will typically be of different strength and in different stages of evolution.

\section{B. OPTICAL CONTINUUM}

The energy released in the annihilation will be degraded by a number of complex processes. Intense local heating of the gas as well as ejection of hot plasma from the exploding stars may give rise to the bright ultra-violet and blue continuum observed in QSOs. Probably synchrotron radiation will contribute even at such short wavelengths because of strong magnetic fields connected with the remnants of the exploding bodies. As such fields must be quite strong to give optical synchrotron radiation, the life-time of the relativistic particles must be correspondingly short. This might explain the rapid light variations often observed in QSOs. Optical variations with longer time scales may be due to the variations in thermal radiation and to variations in the mean activity.

The abovementioned observations of strong and in some cases variable infrared radiation from the QSO 3C 273 and from the nuclei of some Seyfert galaxies and our own galaxy, were interpreted by Low (1970) in terms of a new kind of infrared sources, which he called irtrons. Although there are certain similarities between Low's model and our own, the two models are based on entirely different cosmological hypotheses. Low assumes that matter and antimatter are created in the nuclei of galaxies, whereas Alfvén and myself assume that the galaxies condensed out of a thin ambiplasma. Instead of collisions between mysterious irtrons we speak of collisions between stars of opposite kinds of matter. In both models the annihilation of matter and antimatter will give rise to electrons and positrons with energies around $100 \mathrm{MeV}$. If moving in magnetic fields of the order of $100 \mathrm{G}$, such particles emit synchrotron radiation with the peak in the flux distribution around $4 \times 10^{12} \mathrm{~Hz}$, as observed by Kleinmann and Low for some objects. This radiation will show rapid variations. As mentioned before, such strong fields must be of very small dimensions. In our model they are thought to be confined to a small volume around the exploding stars.

At least in objects where the infrared radiation does not show any rapid intensity variations, part of the infrared energy may come from large dust clouds which are heated to the appropriate temperature by the intense radiation in shorter wavelengths.

\section{OPTICAL LINE EMISSION}

Expansion and cooling of the hot plasma ejected from the exploding stars will lead to the formation of gas clouds emitting broad spectral lines. The existence in the same spectrum of lines originating in gas clouds of very different densities or temperatures is in agreement with our model in which the spectrum will typically be composed of the radiation from several regions, not only inner and outer shells surrounding one explosion but also remnants of many explosions occurring at various times. In addition there will be optical line emission from much larger regions of gas excited by radiation at higher frequencies from the intense source. 


\section{POLARIZATION OF LIGHT}

The variable polarization of light observed in several QSOs (Kinman et al., 1966; Kinman et al., 1968; Elvius, 1968) may be explained as follows. When a smaller body penetrates the outer parts of a star of the opposite kind of matter and is partly annihilated, the explosion will most likely be asymmetric and produce a massive jet of hot plasma, ejected radially from the star. Electron scattering of light from the intense source as well as optical synchrotron radiation may give highly polarized radiation from such a jet. Each new explosion could result in a new more-or-less polarized component of radiation. Thus, sudden changes in the degree of polarization as well as in the position angle could accompany major increases in the intensity of optical radiation.

\section{E. RADIO RADIATION}

In the annihilation process considered in our model, a large fraction $(17 \%)$ of the energy goes into relativistic $(100 \mathrm{MeV})$ electrons and positrons. Although some of these particles may be annihilated immediately, the rest will form a cloud of light ambiplasma, which may leave the star in the form of a jet. This cloud will later continue to expand, as it moves away from the star where it was formed.

Soon after the explosion, however, the relativistic particles of the light ambiplasma will spiral in a magnetic field related to the exploding star. As was mentioned above, such a field may be many orders of magnitude stronger than the general field of the young galaxy. On the other hand it has a very small volume. Thus it seems possible to account for the emission of rather energetic synchrotron radiation without assuming impossibly large energies stored in the magnetic field of the whole QSO.

Our model, therefore, predicts young radio emitters that are extremely small as compared to typical radio sources. Since, we believe, the collisions and explosions take place mainly in the nucleus of the young galaxy, the angular separation of young radio source components should also be quite small, even when the components are due to independent explosions within the same galaxy. The particles will probably, in a later stage, spiral around the lines of force in a larger-scale galactic field, emitting synchrotron radiation at radio wavelengths. Frequencies are probably high in the early stages after the cloud forms, and the energy maximum will shift toward longer wavelengths as the cloud expands and moves away from the active region. We have seen beautiful examples of such shifts demonstrated by several radio astronomers at this symposium. The clouds of relativistic electrons and positrons which comprise the light ambiplasma will drift along the magnetic lines of force. It is possible that in many galaxies a dipole-like magnetic field of large dimensions may exist, along which the light ambiplasma may drift in two opposite directions (each cloud containing both electrons and positrons). In this way the double radio sources which are so often observed may be formed. Immediately following a period of strong activity in the nucleus the new radio source will be concentrated in the nucleus. As time goes on, the two main components of the source become more separated and also become expanded. 
It should perhaps be pointed out that the extended clouds of relativistic particles typical of strong radio galaxies and of some QSOs (which are not among the youngest) according to our model did not originate in a single explosive event but contain the light ambiplasma ejected in many collisions during a period of high activity.

\section{Origin of Elements}

The problem of how the elements were synthesized constitutes a challenge to all cosmologies, and so far none has been able to provide a very satisfactory explanation. If quasi-stellar objects are considered to be very young, the theory of element production by ordinary thermonuclear reactions in stars runs into difficulties because the quasar absorption spectra show the presence of heavy elements. This means that these elements must have been produced no later than the epoch of the quasi-stellar phenomenon.

We have already seen that the violent fluctuations in quasi-stellar object emissions require that stars or starlike bodies of one kind of matter are hit by bodies of the opposite kind of matter. Especially if the hitting bodies are not too big, they will penetrate a star to some depth and cause an explosion at the time of annihilation. A great release of energy will greatly heat a large part of the star and cause a number of shock waves. It is conceivable that heavy elements are produced under such conditions by processes which, in some respect, are similar to those thought to take place in the interior of normal stars and in connection with supernova explosions. The gases ejected from the explosion should then contain newly produced heavy elements. It is likely that some emission and absorption lines in quasars are produced by gases ejected in this manner, when they have cooled down at some distance from the source.

\section{References}

Alfvén, H.: 1965, Rev. Mod. Phys. 37, 652.

Alfvén, H. and Elvius, A.: 1969, Science 164, 911.

Alfvén, H. and Klein, O.: 1963, Arkiv Fysik 23, No. 19.

Colgate, S. A.: 1969, Physics Today 22, 27.

Elvius, A.: 1968, Lowell Obs. Bull. 7, 55.

Field, G. B.: 1964, Astrophys. J. 140, 1434.

Kellermann, K. I., Clark, B. G., Bare, C., Rydbeck, O. E. H., Ellder, J., Hansson, B., Kollberg, E., Höglund, B., Cohen, M. H., and Jauncey, D. L.: 1968, Astron. J. 73, S 101 and Astrophys. J. Letters 153, L209.

Kellermann, K. I. and Pauliny-Toth, I. I. K.: 1969, Astrophys. J. Letters 155, L71.

Kinman, T. D., Lamla, E., and Wirtanen, C. A.: 1966, Astrophys. J. 146, 964.

Kinman, T. D., Lamla, E., Ciurla, T., Harlan, E., and Wirtanen, C. A.: 1968, Astrophys. J. $152,357$.

Kleinmann, D. E. and Low, F. J.: 1970, Astrophys. J. Letters 159, L165.

Low, F. J.: 1970, Astrophys. J. Letters 159, L173. 